\title{
Attosecond analysis
}

\author{
Attosecond spectroscopy promises real-time observation of the motion of electrons inside \\ atoms. Nadya Anscombe talks to Ferenc Krausz from the Max-Planck Institute of Quantum \\ Optics and the Ludwig-Maximilians-University of Munich in Germany about the technology.
}

\section{Why is attosecond} spectroscopy important?

If you want to capture a fast-moving object, you need a fast shutter speed on your camera. In spectroscopy, ultrashort light pulses act as that shutter and can take pictures of ultrafast microscopic dynamics. The development of femtosecond pulses allowed the motion of atoms in molecules to be recorded and led to the birth of femtochemistry. Now, attosecond pulses are required to capture the atomicscale motion of electrons in atoms, molecules or solids. Unlike conventional techniques, such as X-ray crystallography, attosecond spectroscopy gives real-time, direct, dynamic information about the motion of electrons. Indirect ways of accessing such dynamics, such as frequency-domain measurements, work in simple situations (such as in the case of quantum transitions between well isolated states), but if there is interference as a result of the many possible pathways or if many quantum states are involved, the data can be hard to decipher. Attosecond spectroscopy is the only way of getting real-time access to dynamics occurring on the electronic timescale, that is, to processes clocked in the atomic unit of time of 24 as.

\section{What can be achieved with the technology today?}

The shortest pulses demonstrated so far have a duration of 80 as. These pulses, thanks to the order-of-magnitude increase in their photon flux as compared with their predecessors, enable measurements with a dramatically improved signal-to-noise ratio, offering a temporal resolution close to the atomic unit of time. Attosecond spectroscopy was previously used to study isolated atoms in the gas phase, but the technique has now been extended to observe electron motion in condensed-matter systems and on surfaces in real time. Attosecond photoemission spectroscopy presents a clear path towards ultimately uncovering the intermediate processes involved in the ejection of a photoelectron from condensed matter. This is a basic process analysed by Einstein more than a hundred years ago, but with many important details remaining hidden to the present day.

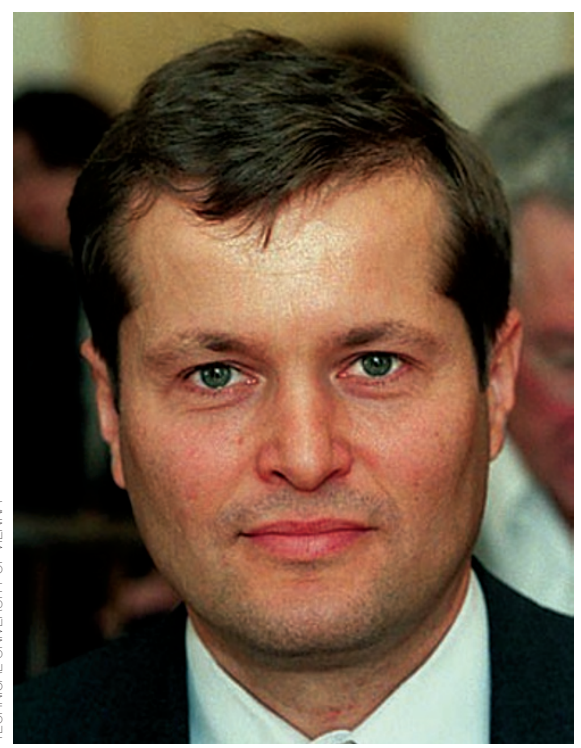

Ferenc Krausz, one of the pioneers of attosecond science.

What are the applications for attosecond spectroscopy?

Because attosecond spectroscopy can be used to look at the dynamic behaviour of electrons, the list of applications is endless. For example, in life sciences attosecond spectroscopy may ultimately create ways of understanding the microscopic origins of how a disease, such as cancer, emerges and develops at the most fundamental level: in terms of the motion of electrons. In biology, structure determines function or malfunction. Although a change in structure can take a long time, the initial event that triggered the change is always an electronic motion. To understand why a molecule decides to change its structure or composition, you have to understand what happens at the instant when that change begins. Attosecond spectroscopy can observe these events in real time.

Advancement of modern electronics towards its ultimate frontier - the atomic scale, in both space and time - means that one day we will want to be able to make electronic circuits from atomic structures and to switch currents in these circuits on and off with the electric field of light. To advance electronics to its ultimate limits we will have to draw on attosecond technology.

Until recently, no experimental technique existed that can directly probe electron-electron interactions in matter on the atomic scale. The accurate theoretical description of these interactions and resultant dynamics becomes very difficult for an increasing number of electrons. Even for systems with just two electrons, no accurate $a b$ initio calculations exist for the dynamical behaviour. Attosecond spectroscopy can, for the first time, offer a method of investigating these interactions experimentally and help theorists to develop simplified but reasonably accurate models for describing multi-electron systems for addressing realistic situations.

There are so many applications for this technology that no one group can investigate them all. That is why it is important that many groups around the world are developing attosecond technology, and I am pleased to see that research in the field is proliferating rapidly.

\section{What needs to be done before the technology becomes commercially available?}

More than half of an attosecond measurement system is already commercially available. Few-cycle phasecontrolled near-infrared laser systems, which serve as a driver of the attosecond pulses, are available off-the-shelf. The rest is simple and complicated at the same time. The attosecond source, a gas jet, is extremely simple, whereas the part needed for performing the attosecond measurements is more complex. The design of a measurement system very much depends on what you want to investigate. There are no major hurdles to be overcome with the technology itself: it is ready to be used. However, the equipment used today is complex and it is important to make it reliable and easy to use, as researchers do not want to go through years of training before they can use the technology to do important research.

Nadya Anscombe is a freelance science and technology journalist based in the UK. 